\title{
Thick and diffuse cisternal clot independently predicts vasospasm-related morbidity and poor outcome after aneurysmal subarachnoid hemorrhage
}

\author{
E. François Aldrich, MD, ${ }^{1}$ Randall Higashida, MD, ${ }^{2}$ Abdel Hmissi, MSc, ${ }^{3}$ Elizabeth J. Le, MD, ${ }^{1}$ \\ R. Loch Macdonald, MD, PhD, ${ }^{4,5}$ Angelina Marr, BSc, ${ }^{3}$ Stephan A. Mayer, MD, ${ }^{6}$ \\ Sébastien Roux, MD, ${ }^{3}$ and Nicolas Bruder, MD ${ }^{7}$
}

\begin{abstract}
'Department of Neurosurgery, University of Maryland, Baltimore, Maryland; '2Department of Neurointerventional Radiology, University of California San Francisco Medical Center, San Francisco, California; ${ }^{3}$ Global Clinical Development, Idorsia Pharmaceuticals Ltd., Allschwil, Switzerland; “Department of Neurological Surgery, University of California San Francisco, Fresno, California; ${ }^{5}$ Department of Surgery, University of Toronto, Ontario, Canada; ${ }^{6}$ Department of Neurology, Henry Ford Neuroscience Institute, Wayne State School of Medicine, Detroit, Michigan; and 'Department of Anesthesia and Critical Care, Hôpital de la Timone, Aix-Marseille Université, Marseille, France
\end{abstract}

OBJECTIVE Aneurysmal subarachnoid hemorrhage (aSAH) is associated with significant morbidity and mortality. The presence of thick, diffuse subarachnoid blood may portend a worse clinical course and outcome, independently of other known prognostic factors such as age, aneurysm size, and initial clinical grade.

METHODS In this post hoc analysis, patients with aSAH undergoing surgical clipping $(n=383)$ or endovascular coiling $(n=189)$ were pooled from the placebo arms of the Clazosentan to Overcome Neurological Ischemia and Infarction Occurring After Subarachnoid Hemorrhage (CONSCIOUS)-2 and CONSCIOUS-3 randomized, double-blind, placebocontrolled phase 3 studies, respectively. Patients without and with thick, diffuse $\mathrm{SAH}$ ( $\geq 4 \mathrm{~mm}$ thick and involving $\geq 3$ basal cisterns) on admission CT scans were compared. Clot size was centrally adjudicated. All-cause mortality and vasospasm-related morbidity at 6 weeks and Glasgow Outcome Scale-Extended (GOSE) scores at 12 weeks after aSAH were assessed. The effect of the thick and diffuse cisternal aSAH on vasospasm-related morbidity and mortality, and on poor clinical outcome at 12 weeks, was evaluated using logistic regression models.

RESULTS Overall, 294 patients (51.4\%) had thick and diffuse aSAH. Compared to patients with less hemorrhage burden, these patients were older (median age 55 vs 50 years) and more often had World Federation of Neurosurgical Societies (WFNS) grade III-V SAH at admission (24.1\% vs 16.5\%). At 6 weeks, all-cause mortality and vasospasmrelated morbidity occurred in $36.1 \%(95 \% \mathrm{Cl} 30.6 \%-41.8 \%)$ of patients with thick, diffuse $\mathrm{SAH}$ and in $14.7 \%(95 \% \mathrm{Cl}$ $10.8 \%-19.5 \%$ ) of those without thick, diffuse SAH. Individual event rates were $7.5 \%$ versus $2.5 \%$ for all-cause death, $19.4 \%$ versus $6.8 \%$ for new cerebral infarct, $28.2 \%$ versus $9.4 \%$ for delayed ischemic neurological deficit, and $24.8 \%$ versus $10.8 \%$ for rescue therapy due to cerebral vasospasm, respectively. Poor clinical outcome (GOSE score $\geq 4$ ) was observed in $32.7 \%$ (95\% Cl 27.3\%-38.3\%) and 16.2\% (95\% Cl 12.1\%-21.1\%) of patients with and without thick, diffuse $\mathrm{SAH}$, respectively.

CONCLUSIONS In a large, centrally adjudicated population of patients with aSAH, WFNS grade at admission and thick, diffuse SAH independently predicted vasospasm-related morbidity and poor 12-week clinical outcome. Patients with thick, diffuse cisternal SAH may be an important cohort to target in future clinical trials of treatment for vasospasm.

https://thejns.org/doi/abs/10.3171/2020.3.JNS193400

KEYWORDS aneurysm; subarachnoid hemorrhage; cerebral vasospasm; vascular disorders

\footnotetext{
ABBREVIATIONS aSAH = aneurysmal SAH; BNI = Barrow Neurological Institute; CONSCIOUS = Clazosentan to Overcome Neurological Ischemia and Infarction Occurring After Subarachnoid Hemorrhage; DCI = delayed cerebral ischemia; DIND = delayed ischemic neurological deficit; GOSE = Glasgow Outcome Scale-Extended; IVH = intraventricular hemorrhage; $\mathrm{SAH}=$ subarachnoid hemorrhage; WFNS = World Federation of Neurosurgical Societies.
}

SUBMITTED December 18, 2019. ACCEPTED March 11, 2020.

INCLUDE WHEN CITING Published online May 22, 2020; DOI: 10.3171/2020.3.JNS193400. 
A NEURYSMAL subarachnoid hemorrhage (aSAH) continues to cause significant morbidity and mortality despite advances in diagnostic and treatment strategies. ${ }^{1,2}$ Cerebral angiographic vasospasm and delayed cerebral ischemia (DCI) or delayed ischemic neurological deficits (DINDs) are well-known sequelae of aSAH that have been identified as leading causes of poor clinical outcomes in this disease. ${ }^{3,4}$ Previous studies have identified factors contributing to vasospasm-related morbidity and mortality, including increased age, history of hypertension, severity of World Federation of Neurosurgical Societies (WFNS) grade, and, most importantly, the extent of intracranial hemorrhage (subarachnoid and intraventricular).$^{5-7}$ Correlation between the amount of SAH detected by CT scan at onset and subsequent development of cerebral vasospasm has been well demonstrated. ${ }^{8-10}$

The Clazosentan to Overcome Neurological Ischemia and Infarction Occurring After Subarachnoid Hemorrhage (CONSCIOUS)-2 and CONSCIOUS-3 trials were conducted between December 2007 and January 2011 at 102 (CONSCIOUS-2) and 106 (CONSCIOUS-3) centers in 27 countries. ${ }^{11,12}$ These randomized, double-blind, placebo-controlled, phase 3 studies aimed to demonstrate that continuous intravenous administration of clazosentan, an endothelin receptor antagonist, would reduce the incidence of cerebral vasospasm-related morbidity events (vasospasm-related cerebral infarction, vasospasm-related DINDs, and the need for rescue therapy) and all-cause mortality at 6 weeks. The trials were identical except that patients in the CONSCIOUS-2 trial had their aneurysms surgically clipped, while in the CONSCIOUS-3 trial, patients had their aneurysms endovascularly treated.

We performed a post hoc analysis of the patients enrolled in the placebo arms of these trials to identify independent risk factors for vasospasm-related morbidity and poor clinical outcome, as these patients may potentially be targeted for future vasospasm-related trials. This large, diverse group of patients with standardized, prospectively collected, centrally adjudicated CT scans, angiograms, and clinical endpoints provided a unique opportunity to better understand the natural history of aSAH. ${ }^{11,12}$ Previous observations of the clinical impact of clot size have been mainly based on data from monocentric rather than international multicentric studies. ${ }^{9}$

Although the modified Fisher scale is widely used, we developed new, more quantitative definitions for differentiating thick versus thin and diffuse versus local hemorrhage in the CONSCIOUS trials in consideration of the key role that SAH clot burden plays in the disease. ${ }^{13} \mathrm{We}$ identified patients with thick, diffuse SAH (defined as $\geq$ $4 \mathrm{~mm}$ thick and involving $\geq 3$ basal cisterns) as a particularly high-risk group with an extensive cisternal clot burden. In this analysis, we sought to determine if this welldefined level of cisternal SAH burden was an independent predictor of death, vasospasm-related morbidity, and poor clinical outcome.

\section{Methods}

\section{Study Population}

For this post hoc analysis, 572 patients from the place- bo arms of the CONSCIOUS-2 and CONSCIOUS-3 trials with SAH caused by a ruptured saccular aneurysm who underwent surgical clipping $(\mathrm{n}=383)$ or endovascular coiling $(n=189)$, respectively, were pooled. The risk of developing vasospasm and vasospasm-related events was expected to be similar with both strategies.$^{14}$ Eligible patients were 18-75 years of age, with a WFNS grade of I-IV prior to the aneurysm-securing procedure that did not worsen to grade $\mathrm{V}$ after the procedure. The CT-documented $\mathrm{SAH}$ was assessed within 48 hours of ictus and was characterized as thick $(\geq 4 \mathrm{~mm}$ on the short axis) or thin $(<4 \mathrm{~mm}$ on the short axis) and diffuse (involving $\geq 3$ basal cisterns) or local (involving 1-2 basal cisterns). Cisterns must have been completely occluded, though larger cisterns (e.g., the sylvian fissure) needed only a significant portion filled. As a result of these criteria, 4 groups of clots were identified: thick/diffuse, thick/local, thin/diffuse, and thin/local. The clinical findings of each group were compared, and the results led to the creation of 2 main groups: 1) thick/diffuse and 2) other types of SAH.

The study sites managed the patients in accordance with published recommendations. ${ }^{1}$ Only drugs and procedures considered standard of care were recommended. Both studies were completed in accordance with the Good Clinical Practice guidelines, the Declaration of Helsinki, and the laws and regulations of the country in which the clinical research was conducted. Written informed consent was obtained for all patients. The CONSCIOUS-2 and CONSCIOUS-3 trials are registered at https://www. clinicaltrials.gov (NCT00558311 and NCT00940095).

\section{Procedures}

Prior to aneurysm treatment, all patients underwent noncontrast CT scanning as well as CT angiography or digital subtraction angiography. Additional CT scans were acquired 12-48 hours after the procedure, at discharge, and 6 weeks after aSAH. Neurological assessments were performed every 6 hours in the intensive care unit and every 12 hours in the general ward until day 14 post-aSAH using the modified Glasgow Coma Scale ${ }^{15}$ and the abbreviated National Institutes of Health Stroke Scale. ${ }^{16,17}$ A CT scan and angiogram were obtained in cases of neurological worsening or symptoms suggestive of cerebral vasospasm or infarction. Patients unable to be neurologically assessed underwent digital subtraction angiography at a mean of $9 \pm 2$ days post-aSAH. Assessment by Glasgow Outcome Scale-Extended (GOSE) ${ }^{8}$ scores was carried out at week 12 by trained centralized interviewers via a structured telephone interview.

Clinical and imaging data were reviewed by a centralized critical events committee, which included an image review committee of neuroradiologists providing independent evaluation of CT scans and angiograms and a clinical review committee of 6 clinicians assessing the occurrence of vasospasm-related morbidity up to week 6 , as well as the contribution of vasospasm to clinical outcome (GOSE score) at week $12 .{ }^{19}$

Vasospasm-related morbidity was defined by at least one of the following events: vasospasm-related cerebral infarction; vasospasm-related DIND (defined as a decrease $\geq 2$ points on the modified Glasgow Coma Scale 
or an increase $\geq 2$ points on the abbreviated National Institutes of Health Stroke Scale lasting for at least 2 hours; or in patients for whom the neurological scales could not be assessed, valid rescue therapy administered for a justifiable reason such as angiographic vasospasm, suspicion of vasospasm on transcranial Doppler or perfusion CT, or clinical signs suggestive of a DIND); or rescue therapy in the setting of confirmed radiological vasospasm. Rescue therapy included the initiation or increase of intravenous vasopressors with or without fluid augmentation, intraarterial vasodilator administration, or balloon angioplasty. Patients treated with vasopressors and fluids must have had a central line and a urinary catheter, and a sustained increase in blood pressure should have occurred. The total volume of all new or worsened cerebral infarcts was also assessed. At week 12, a patient's GOSE score was dichotomized as good $(>4)$ or poor $(\leq 4)$. Mortality included all causes and was derived automatically from the investigator case report form. Patients with thick, diffuse SAH were hypothesized to have a worse clinical course due to more vasospasm-related complications, irrespective of WFNS grade at admission, compared to patients having the other 3 types of SAH burden.

\section{Statistical Analysis}

Data from the placebo arms of the CONSCIOUS-2 and CONSCIOUS-3 trials were pooled and analyzed jointly. Baseline demographics were analyzed using descriptive statistics (all values are means \pm SDs or percentages). The incidence rates are displayed together with the exact $95 \%$ confidence intervals. Normal approximation was used for adjusted relative risk, and exact Clopper-Pearson was used for event indices. In cases of missing assessment for the morbidity component of the primary endpoint or missing information on the vital status, the worst case (i.e., presence of vasospasm-related morbidity or mortality) was assumed. If the GOSE score was not available, a score of 5 (lower moderate disability) was attributed in the absence of clinical evidence for prior neurological impairment, and a score of 3 (lower severe disability) was assigned in any other situation when the patient was alive at week 12. The clot size effect on vasospasm-related morbidity/ mortality and on less favorable 12-week clinical outcome was assessed using logistic regression models adjusting for characteristics at admission. A stepwise selection was performed to identify the most contributive predictors among clot size (thick, diffuse vs other), WFNS grade at admission (I-II vs III-V), age at admission ( $<65$ vs $\geq 65$ years), hydrocephalus at admission (presence vs absence), aneurysm-securing procedure (clip vs coil), sex (male vs female), and aneurysm size ( $>15$ vs $\leq 15 \mathrm{~mm}$ ). A significance level of 0.2 was required for a variable to be allowed into the model and for that variable to stay in the model.

\section{Results}

In this population of 572 patients, baseline demographics were similar between the $51.4 \%$ with and $48.6 \%$ without thick, diffuse SAH (Table 1). Compared to patients with less of a clot burden, patients with thick, diffuse SAH were more likely to present with poor WFNS grade
TABLE 1. Baseline demographics and neurological status on admission

\begin{tabular}{|c|c|c|}
\hline Characteristic & $\begin{array}{l}\text { Thick/Diffuse } \\
\text { Hemorrhage } \\
(n=294)\end{array}$ & $\begin{array}{c}\text { Other } \\
\text { Hemorrhage } \\
(n=278)\end{array}$ \\
\hline \multicolumn{3}{|l|}{ Sex, no. (\%) } \\
\hline Male & $90(30.6)$ & $85(30.6)$ \\
\hline Female & $204(69.4)$ & $193(69.4)$ \\
\hline \multicolumn{3}{|l|}{ Age, yrs } \\
\hline Mean \pm SD & $54.4 \pm 10.7$ & $49.5 \pm 10.8$ \\
\hline Median & 55.0 & 49.5 \\
\hline \multicolumn{3}{|l|}{ Race, no. (\%) } \\
\hline Caucasian/white & $221(75.2)$ & $216(77.7)$ \\
\hline Black & $6(2.0)$ & $10(3.6)$ \\
\hline Asian & $58(19.7)$ & $47(16.9)$ \\
\hline Hispanic & $4(1.4)$ & $4(1.4)$ \\
\hline Other & $5(1.7)$ & $1(0.4)$ \\
\hline \multicolumn{3}{|l|}{ Functional status, no. (\%) } \\
\hline Home, fully independent & $293(99.7)$ & $277(99.6)$ \\
\hline Home, partially independent & $1(0.3)$ & $1(0.4)$ \\
\hline \multicolumn{3}{|l|}{ WFNS admission grade, no. (\%)* } \\
\hline I & $107(36.4)$ & $172(61.9)$ \\
\hline II & $116(39.5)$ & $60(21.6)$ \\
\hline III & $13(4.4)$ & $16(5.8)$ \\
\hline IV & $47(16.0)$ & $28(10.1)$ \\
\hline V & $11(3.7)$ & $2(0.7)$ \\
\hline GCS score & $(n=292)$ & $(n=275)$ \\
\hline $3-6$ & $11(3.8)$ & $2(0.7)$ \\
\hline $7-12$ & $47(16.1)$ & $27(9.8)$ \\
\hline $13-14$ & $127(43.5)$ & $74(26.9)$ \\
\hline 15 & $107(36.6)$ & $172(62.5)$ \\
\hline Admission motor deficit, no. (\%) & $(n=291)$ & $(n=276)$ \\
\hline Absent & $255(87.6)$ & $241(87.3)$ \\
\hline Present & $36(12.4)$ & $35(12.7)$ \\
\hline \multicolumn{3}{|l|}{ Seizure, no. (\%) } \\
\hline Yes & $37(12.6)$ & $20(7.2)$ \\
\hline No & $241(82.0)$ & $250(89.9)$ \\
\hline Unknown & $16(5.4)$ & $8(2.9)$ \\
\hline \multicolumn{3}{|l|}{ Loss of consciousness, no. (\%) } \\
\hline Yes & $136(46.3)$ & $90(32.4)$ \\
\hline No & $150(51.0)$ & $181(65.1)$ \\
\hline Unknown & $8(2.7)$ & $7(2.5)$ \\
\hline
\end{tabular}

GCS = Glasgow Coma Scale.

* Imputed for missing values.

(III-V) (24.1\% vs $16.5 \%)$ and reduced level of consciousness (Glasgow Coma Scale score < 15: 63.4\% vs 37.5\%) on admission. Seizures at onset were observed in $12.6 \%$ of patients with thick, diffuse SAH and in $7.2 \%$ of those without thick, diffuse SAH. Similarly, $46.3 \%$ of patients with thick, diffuse SAH presented with loss of consciousness at ictus compared to $32.4 \%$ of those without (Table 1). Both groups of patients had similar preexisting medical 
TABLE 2. Aneurysm characteristics and CT findings at hospital admission

\begin{tabular}{|c|c|c|}
\hline Characteristic & $\begin{array}{l}\text { Thick/Diffuse } \\
\text { Hemorrhage } \\
(n=294)\end{array}$ & $\begin{array}{c}\text { Other } \\
\text { Hemorrhage } \\
(n=278)\end{array}$ \\
\hline \multicolumn{3}{|c|}{ Repaired aneurysm location, no. (\%) } \\
\hline Supraclinoid ICA & $31(10.5)$ & $26(9.4)$ \\
\hline PCoA & $43(14.6)$ & $28(10.1)$ \\
\hline $\mathrm{ACOA}$ & $124(42.2)$ & $91(32.7)$ \\
\hline $\mathrm{ACA}$ & $17(5.8)$ & $21(7.6)$ \\
\hline MCA & $54(18.4)$ & $90(32.4)$ \\
\hline Posterior circulation or other & $25(9.0)$ & $22(7.9)$ \\
\hline \multicolumn{3}{|l|}{ Aneurysm size, no. (\%) } \\
\hline$\leq 15 \mathrm{~mm}$ & $279(94.9)$ & $267(96.0)$ \\
\hline$>15 \mathrm{~mm}$ & $15(5.1)$ & $11(4.0)$ \\
\hline \multicolumn{3}{|l|}{ Baseline CT findings, no. (\%)* } \\
\hline SAH & $294(100)$ & $276(99.3)$ \\
\hline IVH & $260(88.4)$ & $201(72.3)$ \\
\hline Hydrocephalus $\dagger$ & $201(68.4)$ & $133(47.8)$ \\
\hline Intracerebral hemorrhage & $69(23.5)$ & $60(21.6)$ \\
\hline Extraaxial hematoma & $6(2.0)$ & $4(1.4)$ \\
\hline Midline shift (>5 mm) & $1(0.3)$ & $8(2.9)$ \\
\hline Cerebral infarct & $5(1.7)$ & $3(1.1)$ \\
\hline \multicolumn{3}{|c|}{$\begin{array}{l}\mathrm{ACA}=\text { anterior cerebral artery; } \mathrm{ACOA}=\text { anterior communicating artery; } \mathrm{ICA}= \\
\text { internal carotid artery; } \mathrm{MCA}=\text { middle cerebral artery; } \mathrm{PCOA}=\text { posterior com- } \\
\text { municating artery. } \\
{ }^{*} \text { Findings are not mutually exclusive. } \\
\dagger \text { Bicaudate index > 99th percentile for decile of age. }\end{array}$} \\
\hline
\end{tabular}

comorbidities; however, hypertension was more frequent (51.4\% vs $36.7 \%)$ in patients with thick, diffuse SAH.

Regarding aneurysm location, anterior communicating artery aneurysms accounted for nearly half $(42.2 \%)$ of the $\mathrm{SAH}$ in patients with thick, diffuse $\mathrm{SAH}$, while middle cerebral (32.4\%) and anterior communicating (32.7\%) artery aneurysms caused the majority of SAH in the other patient population (Table 2).

Besides the SAH burden and distribution, baseline CT findings were relatively similar for patients with and without thick, diffuse SAH, though intraventricular hemorrhage (IVH) (88.4\% vs $72.3 \%$ ) and hydrocephalus (68.4\% vs $47.8 \%$ ) were more common in patients with thick, diffuse SAH (Table 2).

Combined all-cause mortality and vasospasm-related morbidity occurred within 6 weeks in $36.1 \%$ (95\% CI $30.6 \%-41.8 \%$ ) of patients with thick, diffuse SAH and in $14.7 \%(95 \%$ CI $10.8 \%-19.5 \%)$ of those without thick, diffuse SAH. The relative risk of experiencing this primary endpoint for patients with thick, diffuse clot was 2.44 (95\% CI 1.77-3.37), and it was similarly significant when adjusted for differences in admission WFNS grade (2.14, 95\% CI 1.57-2.91) (Fig. 1). Individual event rates of vasospasm-related morbidity were also higher for patients with thick, diffuse SAH in all categories: $19.4 \%$ versus $6.8 \%$ for new cerebral infarct, $28.2 \%$ versus $9.4 \%$ for DINDs, and $24.8 \%$ versus $10.8 \%$ for rescue therapy

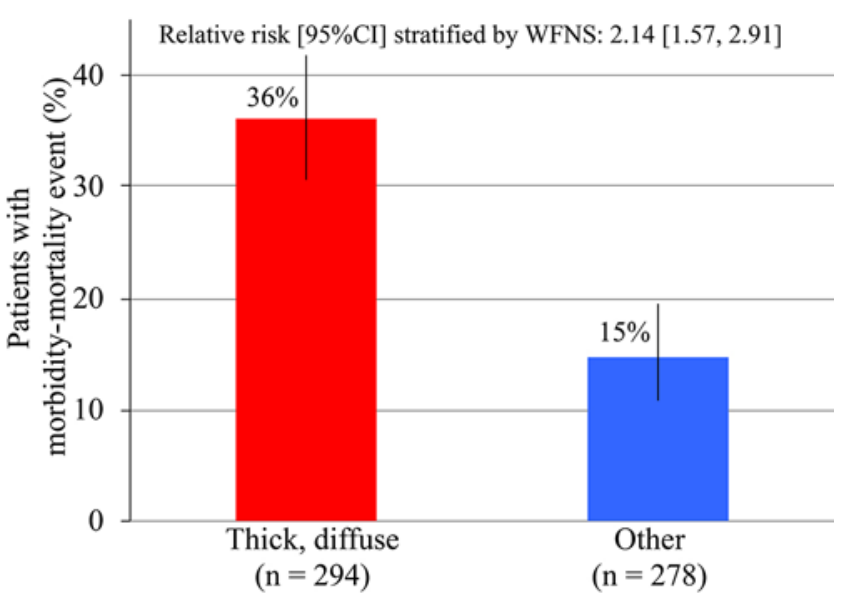

FIG. 1. The effect of hemorrhage burden on morbidity-mortality at 6 weeks. Figure is available in color online only.

due to cerebral vasospasm (Fig. 2). The relative risk for each of these events ranged from 2.30 to 3.02 and again was significant when adjusted for WFNS grade, ranging from 2.19 to 2.62. All-cause mortality itself was more frequent in patients with thick, diffuse SAH (7.5\% vs $2.5 \%)$, with relative risks of 2.97 (95\% CI 1.29-6.85) and 2.20 (95\% CI 0.97-4.99) when adjusted for WFNS grade (Fig. 2). Patients with thick, diffuse SAH suffered mortality from a variety of medical complications not seen in the other group, including sepsis, cardiac failure, hypotension, ileus, and respiratory failure. For both groups, cerebral vasospasm and other brain-related causes of death were the most common causes of mortality (Table 3 ).

At 12 weeks after aSAH, poor clinical outcome, defined by a GOSE score of $\leq 4$, was observed in $32.7 \%$ (95\% CI $27.3 \%-8.3 \%$ ) of patients with thick, diffuse $\mathrm{SAH}$, compared to only $16.2 \%$ (95\% CI $12.1 \%-21.1 \%$ ) of patients with less subarachnoid blood. The relative risks of patients

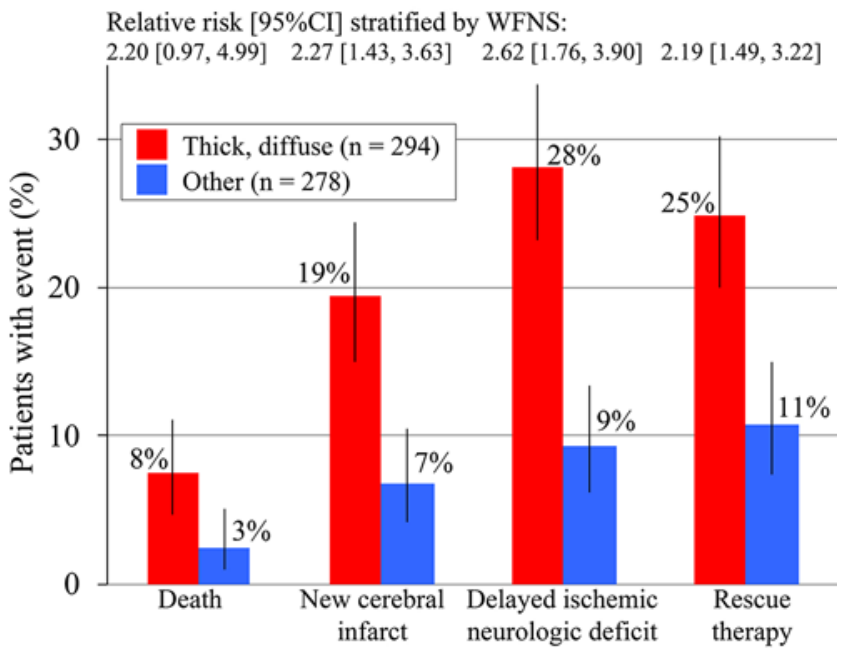

FIG. 2. The effect of hemorrhage burden on vasospasm-related morbidity and all-cause mortality at 6 weeks. Figure is available in color online only. 
TABLE 3. Primary cause of death

\begin{tabular}{lcc}
\hline \multicolumn{1}{c}{ Cause of Death } & $\begin{array}{c}\text { Thick/Diffuse } \\
\text { Hemorrhage } \\
(\mathrm{n}=294)\end{array}$ & $\begin{array}{c}\text { Other } \\
\text { Hemorrhage } \\
(\mathrm{n}=278)\end{array}$ \\
\hline Total no. of deaths (\%) & $25(8.5)$ & $9(3.2)$ \\
\hline Primary cause of death, no. $(\%)^{*}$ & & \\
\hline Cerebral vasospasm & $9(3.1)$ & $4(1.4)$ \\
\hline Cerebral infarction & $11(3.7)$ & $1(0.4)$ \\
\hline Brain edema & $1(0.3)$ & $2(0.7)$ \\
\hline Cerebral hemorrhage & $0(0)$ & $2(0.7)$ \\
\hline Sepsis & $2(0.7)$ & $0(0)$ \\
\hline Cardiac failure & $1(0.3)$ & $0(0)$ \\
\hline Hypotension & $1(0.3)$ & $0(0)$ \\
\hline Ileus & $1(0.3)$ & $0(0)$ \\
\hline IVH & $1(0.3)$ & $0(0)$ \\
\hline Respiratory failure & $1(0.3)$ & $0(0)$ \\
\hline Unknown & $3(1.0)$ & $0(0)$ \\
\hline
\end{tabular}

* Six patients had 2 primary causes of death: 4 patients had cerebral infarction and cerebrovascular vasospasm, 1 patient had cerebral infarction and brain death, and 1 patient had hypotension and sepsis.

with thick, diffuse SAH experiencing a poor clinical outcome were found to be 2.02 (95\% CI 1.47-2.76) and 1.69 (95\% CI 1.23-2.31) when adjusted for admission WFNS grade (Fig. 3).

After stepwise selection, the multivariate logistic regression model included WFNS grade, clot size, and age for the morbidity-mortality model and WFNS grade, clot size, aneurysm size, hydrocephalus, and sex for the clinical outcome model (Table 4). Of the predictors tested, only poor WFNS grade (OR 3.59, 95\% CI 2.30-5.62) and clot size (OR 3.29, 95\% CI 2.15-5.04) were found to be independently associated with both vasospasm-related morbidity and all-cause mortality. A poor WFNS grade (OR 6.11, 95\% CI 3.87-9.66), aneurysm size (OR 3.06, 95\%

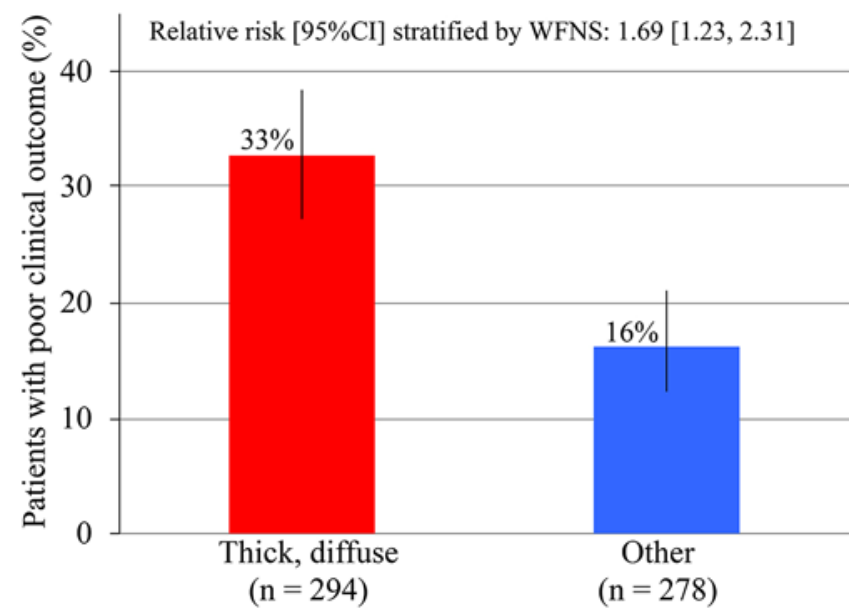

FIG. 3. The effect of hemorrhage burden on clinical outcome at 12 weeks. Poor clinical outcome is defined as a GOSE score of $\leq 4$. Figure is available in color online only.
TABLE 4. Multivariate logistic regression analyses for vasospasm-related morbidity and all-cause mortality at 6 weeks and long-term outcome (GOSE score) at 12 weeks

\begin{tabular}{lcc}
\hline \multirow{2}{*}{$\begin{array}{c}\text { Variables Included } \\
\text { in the Model }\end{array}$} & $\begin{array}{c}\text { Morbidity/Mortality } \\
\text { at } 6 \text { Wks }\end{array}$ & $\begin{array}{c}\text { GOSE Score } \\
\text { at 12 Wks }\end{array}$ \\
\cline { 2 - 3 } $\begin{array}{l}\text { Clot size (thick, diffuse } \\
\text { vs other) }\end{array}$ & $3.29(2.15-5.04)$ & $2.22(1.43-3.45)$ \\
\hline $\begin{array}{l}\text { WFNS grade (III-V } \\
\text { vs I-II) }\end{array}$ & $3.59(2.30-5.62)$ & $6.11(3.87-9.66)$ \\
\hline $\begin{array}{l}\text { Age at admission (<65 } \\
\text { vs } \geq 65 \text { yrs) }\end{array}$ & $1.61(0.87-2.98)$ & - \\
\hline $\begin{array}{l}\text { Aneurysm size (>15 vs } \\
\leq 15 \text { mm) }\end{array}$ & - & $3.06(1.24-7.56)$ \\
\hline $\begin{array}{l}\text { Hydrocephalus (pres- } \\
\text { ence vs absence) }\end{array}$ & - & $1.50(0.95-2.36)$ \\
\hline \begin{tabular}{l} 
Sex (female vs male) \\
\hline
\end{tabular}
\end{tabular}

- = not selected for the multivariate analysis.

* Bicaudate index $>$ 99th percentile for decile of age.

CI 1.24-7.56), and clot size (OR 2.22, 95\% CI 1.43-3.45) were found to be independent predictors of poor 12-week clinical outcome (GOSE score) (Table 4). Of note, sex and hydrocephalus were included in the multivariate analysis for both models, as they have been previously demonstrated to have an impact on outcomes after aSAH. However, in our cohort, they were not found to have a statistically significant impact (Table 4).

Patients with thick, diffuse SAH experienced a greater frequency of treatment-emergent adverse events $(94.9 \%$ vs $86.3 \%$ ). For instance, they had a higher incidence of pneumonia (11.9\% vs 3.6\%) and urinary tract infection $(20.1 \%$ vs $12.9 \%)$. As expected, significantly more patients with thick, diffuse SAH experienced vasospasm (36.7\% vs $18.0 \%)$ and cerebral infarction (12.9\% vs $5.0 \%)$. Hyponatremia ( $22.1 \%$ vs $12.9 \%$ ) was also more common in patients with a larger clot burden (Table 5).

\section{Discussion}

Prevention and treatment of vasospasm-related complications following aSAH have remained frustratingly elusive. Numerous studies have evaluated potential therapies to prevent or treat vasospasm, but few have shown improved long-term neurological outcomes. Prophylactic hypervolemia or angioplasty, and multiple medications, including antiplatelet agents, statins, and magnesium sulfate, have all been trialed but have not shown a clear benefit in clinical outcome..$^{11,20-25}$ Although several trials, such as the prophylactic transluminal balloon angioplasty study by Zwienenberg-Lee et al. and the CONSCIOUS trials with clazosentan by Macdonald et al., demonstrated safety and proof of concept, no benefit was shown in longterm morbidity determined by GOSE score. ${ }^{11,25}$

The lack of success of many promising interventions prompts investigation into the underlying cause for the ongoing failures. Multiple methods to improve aSAH research have been cited, including more unified defini- 
TABLE 5. Summary of treatment-emergent adverse events

\begin{tabular}{lcc}
\hline \multicolumn{1}{c}{ Event } & $\begin{array}{c}\text { Thick/Diffuse } \\
\text { Hemorrhage } \\
(\mathrm{n}=294)\end{array}$ & $\begin{array}{c}\text { Other } \\
\text { Hemorrhage } \\
(\mathrm{n}=278)\end{array}$ \\
\hline Patients w/ at least 1 AE & $279(94.9)$ & $240(86.3)$ \\
\hline Total no. of AEs & 1534 & 1013 \\
\hline Vasospasm & $108(36.7)$ & $50(18.0)$ \\
\hline Pyrexia & $67(22.8)$ & $59(21.2)$ \\
\hline Constipation & $53(18.0)$ & $51(18.3)$ \\
\hline Hyponatremia & $65(22.1)$ & $36(12.9)$ \\
\hline Urinary tract infection & $59(20.1)$ & $36(12.9)$ \\
\hline Hypokalemia & $48(16.3)$ & $35(12.6)$ \\
\hline Anemia & $40(13.6)$ & $27(9.7)$ \\
\hline Cerebral infarction & $38(12.9)$ & $14(5.0)$ \\
\hline Hypertension & $29(9.9)$ & $21(7.6)$ \\
\hline Pneumonia & $35(11.9)$ & $10(3.6)$ \\
\hline Brain edema & $24(8.2)$ & $19(6.8)$ \\
\hline Increased hepatic enzymes & $24(8.2)$ & $19(6.8)$ \\
\hline Hydrocephalus & $24(8.2)$ & $15(5.4)$ \\
\hline Headache & $14(4.8)$ & $24(8.6)$ \\
\hline Nausea & $13(4.4)$ & $24(8.6)$ \\
\hline Increased C-reactive protein & $14(4.8)$ & $16(5.8)$ \\
\hline Increased $\gamma$-glutamyltransferase & $19(6.5)$ & $11(4.0)$ \\
\hline Vomiting & $14(4.8)$ & $15(5.4)$ \\
\hline Other & $238(81.0)$ & $186(66.9)$ \\
\hline HE & &
\end{tabular}

$\mathrm{AE}=$ adverse event.

Values are presented as the number (\%) of patients.

tions and reporting of vasospasm, DCI, imaging results, and clinical outcomes. ${ }^{26}$ There is also an obvious need to develop a sensitive and appropriate clinical endpoint in aSAH that is clinically relevant to clinicians and acceptable to regulators. One method of improving vasospasmrelated trials entails identification of an enriched patient population with a high event rate. Vasospasm is a difficult disease process to study because it does not always develop in patients with aSAH. Report rates of angiographic vasospasm range from $26 \%$ to $66 \%$ and those of DCI range from $20 \%$ to $30 \% .^{14,27-29}$ Furthermore, the severity of angiographic vasospasm does not necessarily correlate with the extent of neurological decline. ${ }^{30}$

Focusing studies on the patient cohorts most likely to experience clinical deterioration due to vasospasm may help demonstrate the benefit of a therapeutic intervention. While the relationship between the amount of SAH shown on CT scans and vasospasm has been previously investigated through varying approaches, $, 10,13,31$ our work provides a distinctive and potentially more accurate view of the natural history of aSAH, given the fact that our cohort is placebo only and, to our knowledge, represents the largest multicentric cohort reported thus far that includes both surgically and endovascularly treated ruptured aneurysms..$^{13}$ Moreover, our data are standardized and were prospectively collected/evaluated with centrally adjudicated CT scans, angiograms, and clinical endpoints.
Our methodology for identifying patients at the highest risk for vasospasm-related morbidity and mortality based on SAH burden as demonstrated on CT scans differs from previous attempts to describe a correlation between the two. For instance, we employed precise clot size definitions rather than the less accurate measurements of common scales such as the classic Fisher scale. In 1980, Fisher et al. first described a grading scale that correlated the amount and distribution of subarachnoid blood with the risk of vasospasm development. ${ }^{32}$ Subsequent investigators, such as Kistler et al. in 1983, Gurusinghe and Richardson in 1984, and Mohsen et al. in 1984, confirmed the relationship between SAH burden and vasospasm by showing that increasing clot size correlated with greater incidence of vasospasm and DCI. ${ }^{33-35}$ More recent studies have sought to refine these findings and have created varying scales to predict the occurrence of vasospasm and DCI. The Hijdra scale quantifies the amounts of blood in 10 basal cisterns and fissures and in 4 ventricles, while the modified Fisher scale adds the presence or absence of intraventricular blood to the original scale. ${ }^{36,37}$ The modified Fisher scale is currently the most commonly used method of grading vasospasm risk in patients with aSAH, and higher grades have been associated with poor long-term outcome. $.^{38} \mathrm{New}-$ ly proposed scales also utilize the thickness of subarachnoid blood in their prediction algorithms. The Barrow Neurological Institute (BNI) has a 1-5 grading scale based on a single measurement of maximum SAH thickness to predict the incidence of symptomatic vasospasm, while the VASOGRADE scale uses modified Fisher grades in combination with WFNS grades to predict DCI. ${ }^{39,40}$

Our method of characterizing SAH on CT scans in order to identify the patient cohort at highest risk of developing vasospasm-related morbidity and mortality could potentially be useful in future studies. We described the hemorrhage with 2 terms: 1) thick or thin and 2) diffuse or local. Categorization of a hemorrhage as diffuse or local was based on the amount of cisternal involvement, where fewer than 3 cisterns was considered local and $\geq 3$ cisterns (including majority occlusion of a major cistern such as the sylvian fissure) was considered diffuse. Although Macdonald et al. utilized a description of SAH as thin/thick and localized/diffuse in their study of factors associated with the development of vasospasm in 2003, our consideration of initial cisternal involvement of blood remains relatively novel. No significant SAH scales, including the modified Fisher scale, account for the number of cisterns filled with subarachnoid blood in addition to the clot thickness, though the Fisher scale has been shown to have prognostic value for vasospasm-related complications. ${ }^{36,41}$

Using our criteria, we identified thick and diffuse SAH as a predictor of not only vasospasm-related morbidity and all-cause mortality at 6 weeks (Figs. 1 and 2), but also poor clinical outcome (GOSE score $\leq 4$ ) at 12 weeks (Fig. 3). These findings mirror those of prior studies. The modified Fisher scale associates the presence of a thick cisternal clot and IVH with an increased risk for DCI, but it has not been consistently predictive of poor clinical outcome. Patients with a modified Fisher score of 3 or 4 (thick SAH without or with IVH) showed greater risk of radiological and symptomatic vasospasm in Kramer and colleagues' com- 
parison of scales evaluating 271 patients. ${ }^{42}$ The BNI scale also stratifies patients according to SAH thickness, but with more even distribution of classes increasing at 5-mm intervals. Patients with thicker SAH clots also had an elevated risk of radiological and symptomatic vasospasm, new cerebral infarction, and poor clinical outcome, with the highest risk demonstrated in patients with $>15$-mm-thick SAH in validation studies including 260 and 1321 patients. ${ }^{40,43,44}$

While our study echoes these findings, the size and standardization of our cohort potentially provide a more generalizable picture of the natural history of the disease, as we examined the association between cisternal clot size burden (as well as other baseline characteristics) and specific manifestations of vasospasm-related morbidity, including new infarcts, DINDs, use of rescue therapy, and clinical outcome, rather than clinical or radiological vasospasm alone. One criticism could be that we did not account for IVH, despite the fact that this has shown prognostic value in several, though not all, studies., ${ }^{75,36,45}$ Our data did include evaluation of IVH presence; however, with $88.4 \%$ and $72.3 \%$ of patients with thick, diffuse and other SAH having IVH, respectively, we felt that further analysis would not be meaningful. Another criticism relates to the fact that patients were included if their WFNS grade was between I and IV prior to the procedure and did not worsen to grade $\mathrm{V}$ postprocedure, which may have limited the enrollment of very severe patients. Finally, although imaging data were assessed by a centralized review committee, no formal assessment of interobserver reliability was performed.

Another interesting observation from our analysis was that the presence or absence of hydrocephalus did not have a statistically significant impact on ultimate 12 -week clinical outcomes. It could be predicted that patients with hydrocephalus present on admission may appear to fare better than those who do not have it since treatment of the hydrocephalus may drastically improve neurological status. However, we found that while these patients improve initially, their 12-week clinical outcomes were not significantly affected by this factor (Table 4).

Furthermore, several investigations have shown that clinical grading scales, such as the Hunt and Hess scale and WFNS scale, may predict neurological outcome better than radiological scales. Dengler et al. demonstrated this by showing that the classic Fisher and BNI scales did not predict clinical outcome as well as the WFNS and Hunt and Hess scales. ${ }^{43}$ Regarding the interaction between SAH clot burden and the risk of DCI, the VASOGRADE scale ascribes the highest risk of DCI to patients with a WFNS grade IV or V regardless of the modified Fisher score. ${ }^{39,43}$ Our analysis showed that thick, diffuse cisternal SAH-as well as WFNS grade at admission-is independently associated with 6-week vasospasm-related morbidity and all-cause mortality and with 12 -week clinical outcome (Table 4).

\section{Conclusions}

The presence of thick and diffuse aSAH (defined as $\geq 4$ $\mathrm{mm}$ thick and involving $\geq 3$ basal cisterns) demonstrated on initial CT scans is associated with an elevated risk of vasospasm-related morbidity and all-cause mortality at 6 weeks as well as poor clinical outcome (a GOSE score of $\leq 4)$ at 12 weeks, even after adjusting for WFNS grade at admission, which was also independently associated with worse outcome. This aSAH subset constitutes an enriched population for vasospasm-related events suitable to test future antivasospastic strategies. Future clinical trials may consider directing attention to this group of patients given their high tendency for vasospasm-related morbidity and mortality.

\section{Acknowledgments}

We thank Emmanuelle Maho of Idorsia Pharmaceuticals Ltd. for her statistical support.

\section{References}

1. Connolly ES Jr, Rabinstein AA, Carhuapoma JR, et al. Guidelines for the management of aneurysmal subarachnoid hemorrhage: a guideline for healthcare professionals from the American Heart Association/American Stroke Association. Stroke. 2012;43(6):1711-1737.

2. Lovelock CE, Rinkel GJ, Rothwell PM. Time trends in outcome of subarachnoid hemorrhage: population-based study and systematic review. Neurology. 2010;74(19):1494-1501.

3. Ayling OG, Ibrahim GM, Alotaibi NM, et al. Dissociation of early and delayed cerebral infarction after aneurysmal subarachnoid hemorrhage. Stroke. 2016;47(12):2945-2951.

4. Macdonald RL. Delayed neurological deterioration after subarachnoid haemorrhage. Nat Rev Neurol. 2014;10(1):44-58.

5. Galea JP, Dulhanty L, Patel HC. Predictors of outcome in aneurysmal subarachnoid hemorrhage patients: observations from a multicenter data set. Stroke. 2017;48(11):2958-2963.

6. Jaja BNR, Saposnik G, Lingsma HF, et al. Development and validation of outcome prediction models for aneurysmal subarachnoid haemorrhage: the SAHIT multinational cohort study. BMJ. 2018;360:j5745.

7. Zanaty M, Nakagawa D, Starke RM, et al. Intraventricular extension of an aneurysmal subarachnoid hemorrhage is an independent predictor of a worse functional outcome. Clin Neurol Neurosurg. 2018;170:67-72.

8. Hijdra A, van Gijn J, Nagelkerke NJ, et al. Prediction of delayed cerebral ischemia, rebleeding, and outcome after aneurysmal subarachnoid hemorrhage. Stroke. 1988;19(10):12501256.

9. Inagawa T. Risk factors for cerebral vasospasm following aneurysmal subarachnoid hemorrhage: a review of the literature. World Neurosurg. 2016;85:56-76.

10. Qureshi AI, Sung GY, Razumovsky AY, et al. Early identification of patients at risk for symptomatic vasospasm after aneurysmal subarachnoid hemorrhage. Crit Care Med. 2000;28(4):984-990.

11. Macdonald RL, Higashida RT, Keller E, et al. Clazosentan, an endothelin receptor antagonist, in patients with aneurysmal subarachnoid haemorrhage undergoing surgical clipping: a randomised, double-blind, placebo-controlled phase 3 trial (CONSCIOUS-2). Lancet Neurol. 2011;10(7):618-625.

12. Macdonald RL, Higashida RT, Keller E, et al. Randomized trial of clazosentan in patients with aneurysmal subarachnoid hemorrhage undergoing endovascular coiling. Stroke. 2012;43(6):1463-1469.

13. Macdonald RL, Rosengart A, Huo D, Karrison T. Factors associated with the development of vasospasm after planned surgical treatment of aneurysmal subarachnoid hemorrhage. J Neurosurg. 2003;99(4):644-652.

14. Charpentier C, Audibert G, Guillemin F, et al. Multivariate analysis of predictors of cerebral vasospasm occurrence 
after aneurysmal subarachnoid hemorrhage. Stroke. 1999;30(7):1402-1408.

15. Darkwah Oppong M, Gembruch O, Herten A, et al. Intraventricular hemorrhage caused by subarachnoid hemorrhage: does the severity matter? World Neurosurg. 2018;111:e693-e702.

16. Schlegel D, Kolb SJ, Luciano JM, et al. Utility of the NIH Stroke Scale as a predictor of hospital disposition. Stroke. 2003;34(1):134-137.

17. Teasdale G, Jennett B. Assessment of coma and impaired consciousness. A practical scale. Lancet. 1974;2(7872):81-84.

18. Wilson JT, Pettigrew LE, Teasdale GM. Structured interviews for the Glasgow Outcome Scale and the extended Glasgow Outcome Scale: guidelines for their use. $J$ Neurotrauma. 1998;15(8):573-585.

19. Macdonald RL, Higashida RT, Keller E, et al. Preventing vasospasm improves outcome after aneurysmal subarachnoid hemorrhage: rationale and design of CONSCIOUS-2 and CONSCIOUS-3 trials. Neurocrit Care. 2010;13(3):416-424.

20. Dorhout Mees SM, van den Bergh WM, Algra A, Rinkel GJ. Antiplatelet therapy for aneurysmal subarachnoid haemorrhage. Cochrane Database Syst Rev. 2007;(4):CD006184.

21. Lennihan L, Mayer SA, Fink ME, et al. Effect of hypervolemic therapy on cerebral blood flow after subarachnoid hemorrhage: a randomized controlled trial. Stroke. 2000;31(2):383-391.

22. Rabinstein AA, Lanzino G, Wijdicks EF. Multidisciplinary management and emerging therapeutic strategies in aneurysmal subarachnoid haemorrhage. Lancet Neurol. 2010;9(5):504-519.

23. Vergouwen MD, Meijers JC, Geskus RB, et al. Biologic effects of simvastatin in patients with aneurysmal subarachnoid hemorrhage: a double-blind, placebo-controlled randomized trial. J Cereb Blood Flow Metab. 2009;29(8):1444-1453.

24. Zhao XD, Zhou YT, Zhang X, et al. A meta analysis of treating subarachnoid hemorrhage with magnesium sulfate. $J$ Clin Neurosci. 2009;16(11):1394-1397.

25. Zwienenberg-Lee M, Hartman J, Rudisill N, et al. Effect of prophylactic transluminal balloon angioplasty on cerebral vasospasm and outcome in patients with Fisher grade III subarachnoid hemorrhage: results of a phase II multicenter, randomized, clinical trial. Stroke. 2008;39(6):1759-1765.

26. Andersen CR, Fitzgerald E, Delaney A, Finfer S. A systematic review of outcome measures employed in aneurysmal subarachnoid hemorrhage $(\mathrm{aSAH})$ clinical research. Neurocrit Care. 2019;30(3):534-541.

27. Frontera JA, Fernandez A, Schmidt JM, et al. Defining vasospasm after subarachnoid hemorrhage: what is the most clinically relevant definition? Stroke. 2009;40(6):1963-1968.

28. Inagawa T, Yahara K, Ohbayashi N. Risk factors associated with cerebral vasospasm following aneurysmal subarachnoid hemorrhage. Neurol Med Chir (Tokyo). 2014;54(6):465-473.

29. Mijiti M, Mijiti P, Axier A, et al. Incidence and predictors of angiographic vasospasm, symptomatic vasospasm and cerebral infarction in Chinese patients with aneurysmal subarachnoid hemorrhage. PLoS One. 2016;11(12):e0168657.

30. Vergouwen MD, Vermeulen M, van Gijn J, et al. Definition of delayed cerebral ischemia after aneurysmal subarachnoid hemorrhage as an outcome event in clinical trials and observational studies: proposal of a multidisciplinary research group. Stroke. 2010;41(10):2391-2395.

31. Frontera JA, Claassen J, Schmidt JM, et al. Prediction of symptomatic vasospasm after subarachnoid hemorrhage: the modified fisher scale. Neurosurgery. 2006;59(1):21-27.

32. Fisher CM, Kistler JP, Davis JM. Relation of cerebral vasospasm to subarachnoid hemorrhage visualized by computerized tomographic scanning. Neurosurgery. 1980;6(1):1-9.
33. Gurusinghe NT, Richardson AE. The value of computerized tomography in aneurysmal subarachnoid hemorrhage. The concept of the CT score. J Neurosurg. 1984;60(4):763-770.

34. Kistler JP, Crowell RM, Davis KR, et al. The relation of cerebral vasospasm to the extent and location of subarachnoid blood visualized by CT scan: a prospective study. Neurology. 1983;33(4):424-436.

35. Mohsen F, Pomonis S, Illingworth R. Prediction of delayed cerebral ischaemia after subarachnoid haemorrhage by computed tomography. J Neurol Neurosurg Psychiatry. 1984;47(11):1197-1202.

36. Claassen J, Bernardini GL, Kreiter K, et al. Effect of cisternal and ventricular blood on risk of delayed cerebral ischemia after subarachnoid hemorrhage: the Fisher scale revisited. Stroke. 2001;32(9):2012-2020.

37. Hijdra A, Brouwers PJ, Vermeulen M, van Gijn J. Grading the amount of blood on computed tomograms after subarachnoid hemorrhage. Stroke. 1990;21(8):1156-1161.

38. Gupta SK, Chhabra R, Mohindra S, et al. Long-term outcome in surviving patients after clipping of intracranial aneurysms. World Neurosurg. 2014;81(2):316-321.

39. de Oliveira Manoel AL, Jaja BN, Germans MR, et al. The VASOGRADE: A simple grading scale for prediction of delayed cerebral ischemia after subarachnoid hemorrhage. Stroke. 2015;46(7):1826-1831.

40. Wilson DA, Nakaji P, Abla AA, et al. A simple and quantitative method to predict symptomatic vasospasm after subarachnoid hemorrhage based on computed tomography: beyond the Fisher scale. Neurosurgery. 2012;71(4):869-875.

41. Klimo P Jr, Schmidt RH. Computed tomography grading schemes used to predict cerebral vasospasm after aneurysmal subarachnoid hemorrhage: a historical review. Neurosurg Focus. 2006;21(3):E5.

42. Kramer AH, Hehir M, Nathan B, et al. A comparison of 3 radiographic scales for the prediction of delayed ischemia and prognosis following subarachnoid hemorrhage. $J$ Neurosurg. 2008;109(2):199-207.

43. Dengler NF, Diesing D, Sarrafzadeh A, et al. The Barrow Neurological Institute Scale revisited: predictive capabilities for cerebral infarction and clinical outcome in patients with aneurysmal subarachnoid hemorrhage. Neurosurgery. 2017;81(2):341-349.

44. Neidert MC, Maldaner N, Stienen MN, et al. The Barrow Neurological Institute Grading Scale as a predictor for delayed cerebral ischemia and outcome after aneurysmal subarachnoid hemorrhage: data from a nationwide patient registry (Swiss SOS). Neurosurgery. 2018;83(6):1286-1293.

45. Jabbarli R, Reinhard M, Roelz R, et al. The predictors and clinical impact of intraventricular hemorrhage in patients with aneurysmal subarachnoid hemorrhage. Int J Stroke. 2016;11(1):68-76.

\section{Disclosures}

Drs. Aldrich, Higashida, Mayer, and Bruder have been consultants/scientific advisors for Idorsia Pharmaceuticals Ltd. and have received consulting fees. Mr. Hmissi, Ms. Marr, and Dr. Roux are employees of Idorsia Pharmaceuticals Ltd. (formerly Actelion Pharmaceuticals Ltd.), which is the owner and developer of clazosentan.

The CONSCIOUS-2 and CONSCIOUS-3 studies were funded by Actelion Pharmaceuticals Ltd. The sponsorship of these studies was transferred to Idorsia Pharmaceuticals Ltd. on July 1, 2018.

\section{Author Contributions}

Conception and design: Aldrich, Hmissi, Marr, Roux. Acquisition of data: Hmissi. Analysis and interpretation of data: Aldrich, Hmissi, Le, Marr, Roux. Drafting the article: Aldrich, Le. Critically revising the article: all authors. Reviewed submitted 
version of manuscript: all authors. Approved the final version of the manuscript on behalf of all authors: Aldrich. Statistical analysis: Hmissi. Administrative/technical/material support: Marr. Study supervision: Roux.

\section{Supplemental Information}

\section{Previous Presentations}

Portions of this work were presented in abstract form at the Vasospasm Congress, Huntington Beach, California, October 2, 2017.

\section{Current Affiliations}

Dr. Le: Department of Neurosurgery, University of Alabama, Birmingham, Alabama.

\section{Correspondence}

E. François Aldrich: University of Maryland, Baltimore, MD. caldrich12@comcast.net. 